\title{
DEFINICIÓN DE LA UTILIDAD DEL MÉTODO DE ARRHENIUS EN EL ESTUDIO DE ESTABILIDAD TÉRMICA DE COMPUESTOS FENÓLICOS EN URERA LACINIATA GOUDOT EXWEDD
}

\section{DEFINITION OF THE UTILITY OF THE ARRHENIUS METHOD INTHE STUDY OF THERMALSTABILITY OF PHENOLIC COMPOUNDS IN URERA LACINIATA GOUDOT EXWEDD}

\begin{abstract}
Suárez Heredia, Martha Azucena Docente Facultad de Ciencias Químicas. Universidad
Central del Ecuador Central del Ecuador
masuarez@uce.edu.ec https://orcid.org/0000-0002-1573-7430
\end{abstract}

Andrade Granja, Consuelo Dolores Docente Facultad de Ciencias Químicas. Universidad Central del Ecuador cdandrade@uce.edu.ec
Villacís Franco, Jhonnathan Gonzalo

Actividad Privada jgvillacis@hotmail.com

\section{RESUMEN}

La degradación térmica de los principios activos naturales es uno de los temas más relevantes en la estabilidad de fitofármacos, por tal motivo es importante determinar: sí una formulación farmacéutica se ajusta al comportamiento de los fármacos de síntesis y, sí el método de determinación del tiempo de vida útil es el adecuado. Con el objetivo de establecer sí, los criterios de zona climática pueden ser aplicables al estudio de estabilidad, mediante el uso de la calorimetría diferencial de barrido, se estandarizó las condiciónes experimentales para la determinación de la temperatura a la cual se degrada el extracto etanólico total de Urera laciniata. Además, bajo estas condiciónes, se definió la influencia de los excipientes sobre la variación de la temperatura de degradación en una forma farmacéutica sólida. Se encontró que, el extracto etanólico seco tiene una temperatura de degradación promedio de $133,17 \pm 0,84^{\circ} \mathrm{C}$ y que los excipientes elevan ésta temperatura en un $54,87 \%$. Para verificar la aplicabilidad de la ecuación de Arrhenius en la evaluación del tiempo de vida útil del fitofármaco, se realizó un estudio de estabilidad a $40^{\circ} \mathrm{C}$ y $75 \%$ de humedad. Mediante la cuantificación de fenoles totales en el extracto etanólico seco y en los comprimidos, se estableció que no existen variaciones significativas que permitan el cálculo de la constante cinética, haciendo inaplicable el método de Arrhenius y el criterio de evaluación mediante zonas climáticas, para la determinación del tiempo de vida media del fitomedicamento.

Palabras clave: urera laciniata; calorimetría diferencial de barrido; extractos vegetales; método de arrhenius.

\section{ABSTRACT}

The thermal degradation of the natural active principles is one of the most relevant topics in the stability of phytopharmaceuticals. For this reason, it is important to determine if a pharmaceutical formulation is adjusted to the behavior of the synthetic drugs and if the method of determining the time of shelf life is adequate. In order to establish whether the climatic zone criteria can be applied to the stability study by using differential scanning calorimetry, the experimental condition for the determination of the temperature at which the total ethanolic extract of Urera laciniata degrades was standardized. Furthermore, under this condition, the influence of the excipients on the variation of the degradation temperature in a solid pharmaceutical form was defined. It was found that the dry ethanolic extract has an average degradation temperature of $133.17 \pm 0.84{ }^{\circ} \mathrm{C}$ and the excipients used raise this temperature 
by $54.87 \%$. To verify the applicability of the Arrhenius equation in the evaluation of the shelf-life of the phytodrug, a stability study was carried out at $40{ }^{\circ} \mathrm{C}$ and $75 \%$ humidity. Through the quantification of total phenols in the dry ethanolic extract and in the pharmaceutical tablets, it was established that there are no significant variations that allow the calculation of the kinetic constant making the Arrhenius method inapplicable for the determination of the half-life time of the phytomedicine, and therefore, evaluation criteria using climatic zones cannot be used either.

Keywords: urera laciniata; differential scanning calorimetry; vegetable extracts; arrhenius method

\section{INTRODUCCIÓN}

Los estudios de estabilidad permiten establecer el período en el cual el medicamento se encuentra en condiciones óptimas para su consumo (ONG Human Info, 2017). Constituyen una serie de pruebas concebidas para obtener información sobre un producto farmacéutico, con el objetivo de definir su tiempo de conservación y su período de vida útil en determinadas condiciones de embalaje o almacenamiento. La estabilidad de los productos farmacéuticos depende de factores ambientales tales como: la temperatura, la humedad y la luz y, de factores relacionados con el producto: las propiedades físico-químicas de la sustancia activa, de los excipientes, la forma farmacéutica, su composición, el proceso de fabricación, la naturaleza del sistema de cierre del envase y las propiedades de los materiales del envase (Organización Mundial de la Salud, 2015).

41 El Consejo Internacional para la Armonización de Requisitos Técnicos para productos farmacéutico $(\mathrm{ICH})$, establece directrices estandarizadas para realizar pruebas que aseguren la calidad, eficacia e inocuidad de productos farmacéuticos, una de esas pruebas son los estudios de estabilidad (ICH, 2017). En Ecuador, el Reglamento para Control de Productos Naturales de Uso Medicinal, vigente desde octubre de 2006, en el Anexo 7 (Ministerio de Salud Publica, 2006) determina que, para obtener el registro sanitario de productos medicinales naturales es necesario: realizar pruebas de estabilidad natural y acelerada, según los criterios de la Organización Mundial de la Salud, OMS.

Los estudios de estabilidad se realizan a partir de la definición del mercado de destino y de las condiciones climáticas, donde van a ser utilizados los fármacos; para ello se ha dividido al mundo en cuatro zonas climáticas (World Health Organization, 2018). Considerando que, de acuerdo a esta división, el Ecuador se encuentra en la zona climática IV, la temperatura y la humedad recomendada para los análisis de estabilidad acelerada en productos farmacéuticos con principios activos relativamente estables es $40 \pm 1^{\circ} \mathrm{C}$ y $75 \pm 5 \%$, repectivamente (Organización Mundial de la Salud, 2015) (World Health Organization, 2018). Estos criterios se aplican también a los estudios de estabilidad de productos naturales de usos medicinal -fitofármacos-. Considerando que los fitofármacos se componen de extractos de plantas o plantas completas, donde el efecto terapéutico lo generan grupos de compuestos que actúan sinérgicamente y que no tienen un comportamiento homogéneo, los estudios de estabilidad deberían considerar estas características.

La industria farmacéutica herbal, se ha incrementado, a nivel mundial, se estima que la producción anual es de 35 mil millones de dólares (Buitron, 1999). Los fitofármacos, al ser productos que provienen de partes de plantas o extractos de estas, están sujetos a factores que pueden inducir un cambio en el producto, ya sea durante el proceso de producción o en el almacenamiento. Los cambios de temperatura, la humedad y la luz pueden ocasionar, principalmente: hidrolisis, oxid hidrólisis, oxidación. racemización e isomerización, con efectos adversos en el fármaco. Los principales problemas ocasionados son: la pérdida en la actividad terapéutica, el cambio de concentración del componente activo, la alteración de la biodisponibilidad y la formación de productos tóxicos (Kumar Sachan \& Kumar, 2015), es importante, por tanto, que este tipo de productos estén sujetos a control. La degrada- 
ción térmica de los principios activos naturales es uno de los temas más relevantes en la estabilidad de fitofármacos, por tal motivo es importante determinar si una formulación farmacéutica se ajusta al comportamiento de los fármacos de síntesis y si el método de determinación del tiempo de vida útil es el adecuado

La calorimetría diferencial de barrido (DSC) permite, entre otros análisis, definir las temperaturas de descomposición y la estabilidad térmica de: sólidos compactos, plásticos, gomas, resinas, otros materiales orgánicos, cerámicos, vidrios, mezclas complejas, polvos, muestras viscosas, líquidos, etc. (Zambrano, et al., 2017) (Bajaj, Singla, \& Sakhuja, 2012), entre los cuales se encuentran las formulaciones farmacéuticas de origen natural. Por sus características, esta metodología utiliza pequeñas cantidades de muestra y puede proporcionar información de la temperatura a la que se degradan los componentes de las muestras vegetales y ser un aporte para los estudios de estabilidad, con datos certeros, que no dependan exclusivamente de las condiciones de una zona climática definida.

Para el estudio se seleccionó Urera laciniata Goudot ex Wedd o también Ilamada ortiga, que es conocida desde la antigüedad por los habitantes de la región amazónica del Ecuador y su información etnobotánica refiere usos contra: enfermedades reumáticas, malaria, fiebre y dolores musculares (Luziatelli, Sørensen, Theilade, \& Mølgaard, 2010), también se reportan usos como analgésico (Ríos, Koziol, Borgtoft, \& Granda, 2007). Las especies de esta familia -Urticaceae- comparten los mismos principios activos: el ácido fórmico y los flavonoides, responsables de las propiedades antiinflamatorias que se les atribuyen (Olivo \& Pazmiño, 2013). La literatura revisada no reporta la existencia de datos de estabilidad de extractos y/o formas farmacéuticas, elaboradas con esta especie. Adicionalmente, Urera laciniata posee un grupo de principios activos -los compuestos fenólicos- que tienen una amplia actividad terapéutica y, estudiados podrían configurar una metodología utilizable en otras variedades vegetales que los contengan.

\section{METODOLOGÍA}

Se colectó Urera laciniata Goudot ex Wedd, en el Cantón Archidona - Provincia de Napo- la identificación botánica fue realizada en el Herbario $Q$ de la Universidad Central del Ecuador.

Las hojas de la planta fueron lavadas con agua destilada tipo III y desinfectadas con permanganato de potasio 0,0001\%. El material vegetal se secó en una estufa por convección forzada a $40 \pm 1^{\circ} \mathrm{C}$, hasta humedad relativa inferior al 10\%. La muestra se sometió a molienda en un molino manual y tamizaje en un Tamiz Humboldt \# 30. El material vegetal se desengrasó con hexano analítico. Los compuestos fenólicos se extrajeron por percolación a 20 gotas/min con etanol analítico 96\% (Suárez \& Narváez, 2016).

Con el extracto total se elaboraron comprimidos, mediante granulación húmeda (Suárez \& Mora, 2016), utilizando como excipientes una mezcla de $85,52 \%$ de lactosa, 0,66\% de aerosil, $0,66 \%$ de estearato de magnesio, $1,32 \%$ de almidón y $11,84 \%$ de extracto etanólico seco. La composición utilizó la fórmula unitaria desarrollada experimentalmente, en los laboratorios FARCOL de la ciudad de Quito.

Se identificaron cualitativamente los compuestos fenólicos, tanto en el extracto como en los comprimidos, por reacción con cloruro férrico $5 \%$. Se determinó la presencia de ácido cafeico por cromatografía de capa fina de alto rendimiento (HPTLC), utilizando un estándar analítico de ácido cafeico Sigma-Aldrich, CAS: 331-39-5. El desarrollo cromatográfico utilizó como fase estacionaria placas Macherev-Nagel, nanosil C18-100/UV254 y como fase móvil tolueno: hexano:metanol $(17,5: 17,5: 5,0)$. La cuantificación de fenoles totales expresados como ácido cafeico, se realizó espectrofotométricamente por el método de Folin- Ciocalteau (Espinosa, Garzón, \& Medina, 2016), en un equipo Varian BioCary50.

La definición de las condiciones para el corrido calorimétrico se realizó con un diseño experimental 22, aleatorizado en el programa JMP Statical Discovery® a un 95\% de confianza. La variable respuesta analizada fue la temperatura degradación de la mezcla de compuestos (Ti). 
Los factores y niveles de estudio se presentan en la tabla 1

Tabla 1. Factores y niveles de estudio para extracto etanólico y comprimidos de Urera laciniata Goudot ex Wedd

\begin{tabular}{|l|l|r|r|}
\hline \multicolumn{1}{|c|}{ Factores } & \multicolumn{1}{|c|}{$\begin{array}{c}\text { Código } \\
\text { /unidades }\end{array}$} & \multicolumn{2}{|c|}{ Niveles $^{*}$} \\
\hline $\begin{array}{l}\text { Velocidad de } \\
\text { la rampa de } \\
\text { temperatura } \\
\begin{array}{l}\text { Peso de } \\
\text { muestra }\end{array}\end{array}$ & RT $\left({ }^{\circ} \mathrm{C} / \mathrm{min}\right)$ & 10 & 20 \\
\hline
\end{tabular}

* Los signos positivo y negativo corresponden a los niveles superior e inferior de los factores estudiados.

Se corrieron termogramas del extracto y los comprimidos para definir su comportamiento térmico, en el día cero y día sesenta del análisis de estabilidad de fenoles, en un Calorímetro de Barrido Diferencial, DSC Q 2000, calibrado con Indio para un rango de temperatura de $0-300^{\circ} \mathrm{C}$, y con un flujo de nitrógeno de $25 \mathrm{~mL} / \mathrm{min}$. Se utilizaron cápsulas de aluminio selladas tipo A.

Se realizaron pruebas de estabilidad para los comprimidos, en sesenta días de almacenamiento a $40^{\circ} \mathrm{C}$ y $75 \%$ de humedad relativa, se analizó la concentración de fenoles totales.

\section{DISCUSIÓN}

En la muestra seca de Urera laciniata se obtuvo una concentración, en peso, de extracto etanólico total correspondiente a 9,88 \pm $0,86 \%$. Se identificó cualitativamente la presencia de compuestos fenólicos y

la separación de ácido cafeico a un $\mathrm{Rf}$ de 0,88 coincidente con el estándar; en las condiciones experimentales definidas. La cuantificación de fenoles totales, expresada como ácido cafeico, dio un resultado de 145,43 $\pm 7,46$ $\mathrm{mg}$ de fenoles/g extracto seco y de 1430,93 \pm $88,03 \mathrm{mg}$ fenoles totales/100g muestra seca. A partir del extracto etanólico se elaboraron comprimidos, mediante granulación húmeda, con un peso promedio de 443,00 $\pm 3,18 \mathrm{mg}$ y una concentración de fenoles totales de 7,75 $\mathrm{mg} /$ tableta que, mediante pruebas preliminares, se definió, se encuentra en el límite de detección del espectrofotómetro utilizado.

La definición de las condiciones de corrido de los termogramas, se realizó mediante el aná- lisis de los datos de temperatura de degradación promedio, para el extracto etanólico y los comprimidos que se presentan en la tabla 2.

Tabla 2. Temperaturas de degradación promedio en el estudio térmico de extracto etanólico y comprimidos de Urera laciniata Goudot ex Wedd

\begin{tabular}{|l|c|c|c|c|}
\hline \multirow{2}{*}{$\mathbf{N}^{\circ}$} & \multicolumn{2}{|c|}{ VARIABLES } & \multirow{2}{*}{ EXTRACTO } & COMPRIMIDOS \\
\cline { 1 - 2 } & $\begin{array}{c}\mathbf{R T}\left({ }^{\circ} \mathbf{C} /\right. \\
\mathbf{m i n})\end{array}$ & $\begin{array}{c}\mathbf{P M} \\
\mathbf{( m g )}\end{array}$ & $\mathbf{T}^{\circ} \mathbf{C}$ & T'C \\
\hline 1 & - & - & 132,91 & 202,94 \\
\hline 2 & + & - & 132,75 & 204,15 \\
\hline 3 & - & + & 134,41 & 208,79 \\
\hline 4 & + & + & 132,60 & 207,67 \\
\hline
\end{tabular}

El cálculo de la magnitud de efectos ocasionados por los factores de estudio: velocidad de la rampa de temperatura (RT) y peso de muestra (PM) sobre la variable respuesta, temperatura de degradación; se realizó mediante el algoritmo de Yates. Los valores de la magnitud de los efectos, así como su significancia estadística se muestran en la tabla 3, para el extracto etanólico y, en la tabla 4 para los comprimidos.

Tabla 3. Magnitud y significancia estadística de los efectos de la variación de la velocidad de la rampa de temperatura (RT) y el peso de muestra (PM) sobre la temperatura de degradación de extracto etanólico de Urera laciniata Goudot ex Wedd

\begin{tabular}{|l|l|l|l|l|}
\hline \multicolumn{2}{|l|}{ VARIABLES } & \multicolumn{3}{l|}{ EXTRACTO } \\
\hline $\begin{array}{l}\text { RT } \\
\left({ }^{\circ} \mathbf{C} / \mathbf{m i n}\right)\end{array}$ & $\begin{array}{l}\text { PM } \\
(\mathbf{m g})\end{array}$ & $\begin{array}{l}\text { VALOR } \\
\text { EFECTO }\end{array}$ & $\begin{array}{l}\text { IDENTIFI- } \\
\text { CACION }\end{array}$ & SIGNIFICANCIA \\
\hline- & - & 132,17 & Media & \\
+ & - & $-0,49$ & RT & no significativo \\
- & + & 0,17 & PM & no significativo \\
+ & + & $-0,33$ & $(\mathrm{RT})(\mathrm{PM})$ & no significativo \\
\hline
\end{tabular}

Tabla 4. Magnitud y significancia estadística de los efectos de la variación de la velocidad de la rampa de temperatura (RT) y el peso de muestra (PM) sobre la temperatura de degradación de comprimidos, en base de Urera laciniata Goudot ex Wedd

\begin{tabular}{|c|c|c|c|c|}
\hline \multicolumn{2}{|c|}{ VARIABLES } & \multicolumn{3}{|c|}{ COMPRIMIDOS } \\
\hline $\begin{array}{c}\text { RT } \\
\text { ( }{ }^{\circ} \mathbf{C} / \\
\text { min) }\end{array}$ & $\begin{array}{c}\text { PM } \\
(\mathbf{m g})\end{array}$ & $\begin{array}{c}\text { VALOR } \\
\text { EFECTO }\end{array}$ & $\begin{array}{c}\text { IDENTIFI- } \\
\text { CACION }\end{array}$ & SIGNIFICANCIA \\
\hline- & - & 205,89 & Media & \\
+ & - & 0,025 & RT & no significativo \\
- & + & 4,705 & PM & no significativo \\
+ & + & $-1,14$ & $(\mathrm{RT})(\mathrm{PM})$ & no significativo \\
\hline
\end{tabular}


De acuerdo con los datos estadísticos presentados en las tablas 3 y 4 , el incremento de la velocidad de la rampa de temperatura (RT), utilizada en el calorímetro y el peso de muestra (PM), utilizada para el desarrollo de los termogramas, en extracto etanólico seco y comprimidos elaborados con Urera laciniata Goudot ex Wedd, son estadísticamente no significativos, por tanto no tienen incidencia en el estudio. Para la elaboración de los termogramas se seleccionaron los niveles más bajos de los factores de estudio, que implican menor consumo de recursos y estos son: $10^{\circ} \mathrm{C} / \mathrm{min}$ y $0,5 \mathrm{mg}$ de muestra.

La temperatura de degradación media para el extracto etanólico es de $132,17 \pm 0,84^{\circ} \mathrm{C}$ y para el caso de los comprimidos es 205,89 \pm $2,79^{\circ} \mathrm{C}$. Los excipientes utilizados estabilizan al extracto etanólico incrementando su temperatura de degradación en $72,94^{\circ} \mathrm{C}$. Se observa que existe un efecto térmico de estabilidad cuando se añaden los excipientes en el extracto, ya que la temperatura de degradación de la forma farmacéutica se encuentra desplazada hacia valores más altos, tanto en el caso del extracto (Tabla 4), como en el de los excipientes (Tabla 5). Esta estabilización podría deberse a las fuerzas de interacción generadas entre los grupos polares de los excipientes, especialmente la lactosa, con los grupos $\mathrm{OH}$ de los compuestos fenólicos, en forma de glicósidos de la especie vegetal analizada (Olivo \& Pazmiño, 2013), (PineIli, Ieri, Vignolini, Bacci, Baronti, \& Romani, 2008).

Tabla 5. Resultados de degradación de excipientes en el estudio térmico de comprimidos, en base de

Urera laciniata Goudot ex Wedd

\begin{tabular}{|l|r|}
\hline \multicolumn{1}{|c|}{ EXCIPIENTE } & \multicolumn{2}{|c|}{$\begin{array}{c}\text { TEMPERATURA } \\
\text { DEGRADACIÓN }{ }^{\circ} \mathbf{C}\end{array}$} \\
\hline Lactosa & 146,69 \\
Almidón & 82,16 \\
Estearato de magnesio & 93,35 \\
Aerosil & No presenta pico en el rango \\
& analizado \\
\hline
\end{tabular}

Lo anteriormente expuesto se verifica, analizando los termogramas presentados en las figuras 1 y 2. La mezcla de excipientes en las proporciones utilizadas en la forma farmacéutica presenta dos picos definidos en $146^{\circ} \mathrm{C}$ y $217^{\circ} \mathrm{C}$, que no son coincidentes con los picos obtenidos para los comprimidos y el extracto etanólico

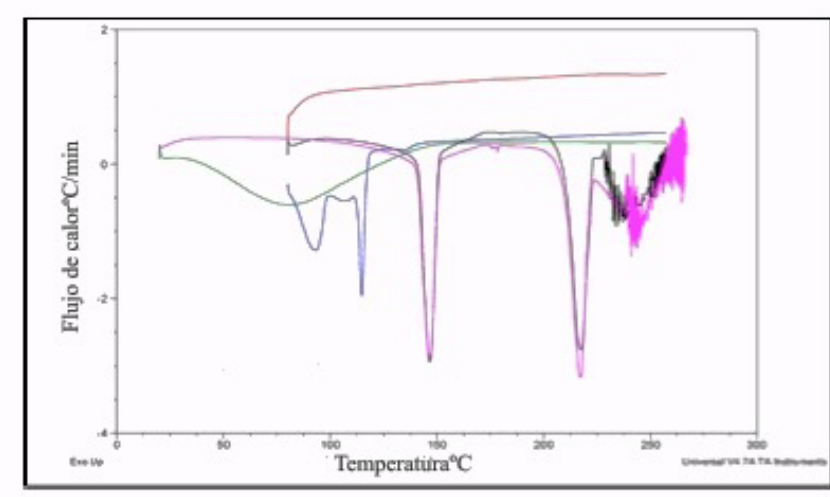

Figura 1. Termogramas de excipientes. Aerosil (rojo), almidón (verde), estearato de magnesio (azul), mezcla (rosado)

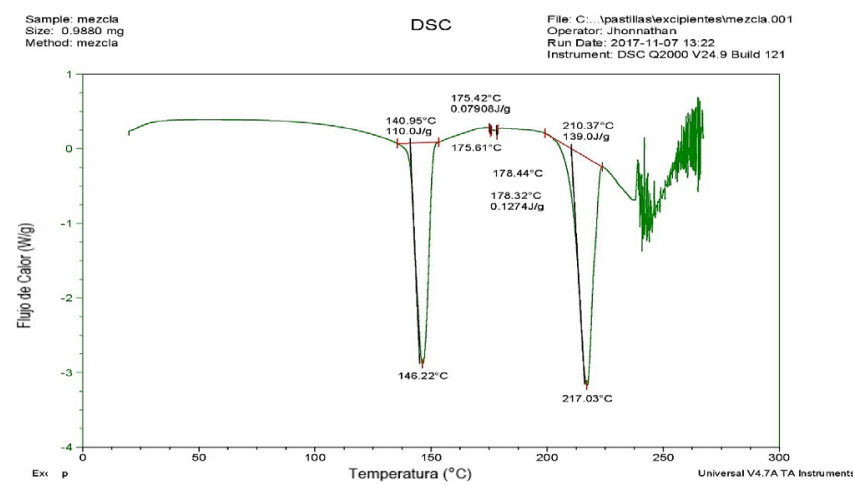

Figura 2. Termograma de la mezcla de excipientes utilizada en la formulación: aerosíl, estearato de magnesio, almidón y lactosa.

De acuerdo con los resultados obtenidos que muestran que la degradación térmica del extracto y de los comprimidos, se da a temperaturas superiores a las utilizadas en las condiciones a las cuales se realizan las pruebas de estabilidad, éstas no serían aplicables para el caso de los comprimidos de esta especie vegetal. Para verificar la aplicabilidad de las condiciones sugeridas para las pruebas de restabilidad por la OMS (2015), se tomaron como referencia los parámetros dados para la zona climática IV (Organización Mundial de la Salud, 2015) .

Los resultados de los termogramas que indican las temperaturas de degradación para los comprimidos, al cabo de sesenta días de almacenamiento a $40^{\circ} \mathrm{C}$, a una humedad relativa de $75 \%$ y en diferentes tipos de envase, se presentan en la tabla 6 . Adicionalmente, se verifico la influencia del tipo de envase en la estabilidad térmica. 
Tabla 6. Temperaturas de degradación de comprimidos en $\mathbf{6 0}$ días de almacenamiento a $40^{\circ} \mathrm{C}$ y humedad relativa de $75 \%$ en el estudio térmico de comprimidos en base de Urera laciniata Goudot ex Wedd

\begin{tabular}{|c|l|c|}
\hline $\begin{array}{c}\text { TEMPERATURA } \\
\text { ALMACENAMIENTO } \\
{ }^{\circ} \mathrm{C}\end{array}$ & $\begin{array}{c}\text { ESPECIFICACIONES } \\
\text { ENVASE }\end{array}$ & $\begin{array}{c}\text { TEMPERATURA } \\
\text { DEGRADACIÓN } \\
{ }^{\circ} \mathbf{C}\end{array}$ \\
\hline 40 & $\begin{array}{l}\text { Ambar, polietilén } \\
\text { tereftalato(PET) alta } \\
\text { densidad }\end{array}$ & 206,18 \\
\hline 40 & $\begin{array}{l}\text { Blanco, polietilén } \\
\text { tereftalato (PET) alta } \\
\text { densidad }\end{array}$ & 207,08 \\
\hline 40 & $\begin{array}{l}\text { Transparente, poli- } \\
\text { etilén tereftalato } \\
\text { (PET) altadensidad }\end{array}$ & 206,82 \\
\hline
\end{tabular}

De acuerdo con los datos presentados en la tabla 6 , se puede establecer que la temperatura de degradación no tiene un cambio significativo, debido al tiempo de almacenamiento. Se puede establecer que el tipo de envase utilizado en el almacenamiento de los comprimidos en un tiempo de 60 días, no influye en la temperatura de degradación, ya que se tienen diferencias que corresponden a valores que se encuentran entre el 0,1-1,0\%, respecto de la temperatura de degradación inicialmente determinada.

La estabilidad de los comprimidos se controló mediante la cuantificación espectrofotométrica de fenoles totales, en el tiempo de almacenamiento de 60 días, considerando la influencia del tipo de envase utilizado; los valores determinados para la concentración de fenoles se presentan en la tabla 7 .

Tabla 7. Concentración de fenoles totales en comprimidos, en 60 días de almacenamiento a $40^{\circ} \mathrm{C}$ y humedad relativa de $75 \%$, en el estudio de estabilidad comprimidos en base de Urera laciniata Goudot ex Wedd

\begin{tabular}{|l|c|c|c|}
\hline ENVASE & TRANSPARENTE & BLANCO & AMBAR \\
$\mathbf{m g}$ & $\begin{array}{c}\mathbf{m g} \text { fenoles/ } \\
\text { tableta }\end{array}$ & $\begin{array}{c}\text { mg fenoles/ } \\
\text { tableta }\end{array}$ \\
\hline 0 & 7,26 & 7,26 & 7,26 \\
5 & 6,80 & 7,24 & 6,87 \\
10 & 6,87 & 6,97 & 7,34 \\
12 & 6,02 & 7,15 & 7,21 \\
14 & 6,83 & 6,51 & 6,76 \\
17 & 6,64 & 6,52 & 6,38 \\
20 & 6,60 & 6,44 & 6,26 \\
24 & 7,06 & 7,08 & 7,15 \\
\hline
\end{tabular}

\begin{tabular}{|c|c|c|c|}
\hline 27 & 6,84 & 7,05 & 6,89 \\
\hline 31 & 7,03 & 7,09 & 6,55 \\
\hline 46 & 6,98 & 6,67 & 6,68 \\
\hline 60 & 7,36 & 7,14 & 7,44 \\
\hline Medias & $6,86 \pm 0,35$ & $6,92 \pm 0,30$ & $6,89 \pm 0,39$ \\
\hline \multicolumn{4}{|c|}{$\begin{array}{l}\text { En la cuantificación de compuestos fenólicos } \\
\text { totales, durante el almacenamiento, los cam- } \\
\text { bios de concentración en cada tipo de frasco } \\
\text { en } 60 \text { días de almacenamiento no son signifi- } \\
\text { cativos de acuerdo a la dispersión de datos } \\
\text { respecto de sus medias. }\end{array}$} \\
\hline
\end{tabular}

\section{CONCLUSIONES}

El estudio térmico de extractos vegetales, mediante calorimetría diferecial de barrido, aporta información valiosa respecto de la degradación de los componentes químicos de los extractos vegetales y las formas farmacéuticas, elaborados con estos extractos. La composición química particular de cada especie vegetal hace necesario que se considere que la degradación no se da a una temperatura fija para todos los casos, sino que existen variantes propias para cada especie, que deben ser estudiadas. Se observa que para Urera laciniata Goudot ex Wedd, la alta temperatura de degradación del extracto y de los comprimidos; así como tambien los datos de concentración obtenidos en el análisis del marcador fitoquímico en Urera laciniata Goudot ex Wedd, evidencian una ausencia de variación, lo que hace inviable la determinación del tiempo de vida media de los comprimidos, utilizando el método de Arrhenius y la aplicación del criterio de uso de las condiciones de Zona Climática, para evaluar la estabilidad del marcador fitoquímico en esta especie. Se deberían analizar otras alternativas como la verificación de la presencia de los componentes activos, mediante perfiles cromatográficos elaborados, tanto con los extractos como con las formas farmacéuticas desarrolladas (Suárez \& Venegas, 2016).

\section{AGRADECIMIENTO}

Los autores expresan su agradecimiento al Señor Luis Cola, Gerente de FARCOL LABORATORIOS, quien nos brindó su apoyo para la elaboración, sin costo, de las formas far- 
macéuticas que se utilizaron en el presente estudio.

\section{REFERENCIAS}

Bajaj, S., Singla, D., \& Sakhuja, N. (2012). Stability Testing of Pharmaceutical Products. Journal of Applied Pharmaceutical Science, 129-138.

Buitrón, X. (1999). Uso y comercialización de plantas medicinales: Situación actual y aspectos importantes para su conservacion. Cambridge: TRAFFIC International.

Espinosa, W., Garzón, L., \& Medina, O. (2016). Validación de una metodología para cuantificación de polifenoles totales, en procesos de extracción asistida por microondas sobre frutos de la especie colombiana Vaccidium meridionale. Revista colombiana de ciencoas químico framacéuticas , 45 (1), 109 - 126.

IC. (2017). ICH harmonisation for better healt. Recuperado el 29 de enero de 2017, de https://www.ich.org/products/guidelines/quality/article/quality-guidelines.html

Kumar Sachan, A., \& Kumar, A. (2015). Stability testing of herbal products. Journal of Chemical and Pharmaceutical Research , 511514.

Luziatelli, G., Sørensen, M., Theilade, I., \& Mølgaard, P. (2010). Asháninka medicinal plants: a case study from the native community of Bajo Quimiriki, Junín, Peru. Journal of Ethnobiology and Ethnomedicine .

Ministerio de Salud Publica. (26 de octubre de 2006). Reglamento y control de productos naturales de uso medicinal. 385 . Quito, Ecuador: Registro Oficial.

Olivo, S., \& Pazmiño, J. (2013). Estudio comparativo de la utilización de plantas medicinales durante el parto tradicional por organizaciones de parteras de Otavalo y Loreto 2016. Ibarra, Ecuador: Universidad Técnica del Norte.

ONG Human Info. (10 de Febrero de 2017). Medicamentos Esenciales y Productos de Salud de la OMS. Obtenido de http://apps.who.
int/medicinedocs/es/d/Jh2976s/\#Jh2976s.1

Organización Mundial de la Salud, O. (2015). Comité de expertos en especificaciones para preparaciones farmacéuticas. OMS.

Pinelli, P., leri, F., Vignolini, P., Bacci, L., Baronti, S., \& Romani, A. (2008). Extraction and HPLC Analysis of Phenolic Compounds in Leaves, Stalks, and Textile Fibers of Urtica dioica L. Journal of Agricultural and Food Chemistry, 9127-9132.

Ríos, M., Koziol, M., Borgtoft, H., \& Granda, G. (2007). Plantas útiles del Ecuador. Aplicaciones, retos y perspectivas (Quinta edición ed.). Quito, Ecuador: Abya-Yala.

Suárez, M., \& Mora, S. (2016). Procedimientos operativos estándar para formas farmacéuticas sólidas. Saarburken, Alemania: Académica Española-OmniScriptumGmbH.

Suárez, M., \& Narváez, G. (2016). Copigmentación intermolecular de antocianinas glicosiladas. Saarbrucken, Alemania: Académica EspañolaOmnScriptum GmbH.

Suárez, M., \& Venegas, A. (2016). Método cromatográfico HPTLC para control de fitomedicamentos. Revista de la Facultad de Ciencias Médicas, 91

World Health Organization. (2018). Essential Medicines and Health Products Information Portal. Recuperado el 5 de 12 de 2018, de http://apps.who.int/medicinedocs/en/d/ Js5516e/15.3.html

Zambrano, A., Castellar, G., Vallejo, W., Piñeres, I., Cely, M., y otros. (julio de 2017). Conceptual approach to thermal analysis and its main applications. Prospectiva . 